\title{
Synchronization of MEMS Resonators and Mechanical Neurocomputing
}

\author{
Frank C. Hoppensteadt, Member, IEEE, and Eugene M. Izhikevich
}

\begin{abstract}
We combine here two well-known and established concepts: microelectromechanical systems (MEMS) and neurocomputing. First, we consider MEMS oscillators having low amplitude activity and we derive a simple mathematical model that describes nonlinear phase-locking dynamics in them. Then, we investigate a theoretical possibility of using MEMS oscillators to build an oscillatory neurocomputer having autocorrelative associative memory. The neurocomputer stores and retrieves complex oscillatory patterns in the form of synchronized states with appropriate phase relations between the oscillators. Thus, we show that MEMS alone can be used to build a sophisticated information processing system (U.S. provisional patent 60/178,654).
\end{abstract}

Index Terms-Andronov-Hopf bifurcation, $\mu$ resonantors, neural networks, oscillatory associative memory, smart matter.

\section{INTRODUCTION}

$\mathbf{M}$ ICROELECTROMECHANICAL systems (MEMS) are used to create miniature, highly accurate sensors and actuators which can gather nonelectronic information from the physical world, such as temperature, barometric pressure, relative humidity, acceleration, vibration, etc. [15], [19]. This analog information may be digitized and transmitted to a resident microcontroller for signal processing. When sophisticated signal analysis is required, such as extraction of spatio-temporal patterns, the signal may be radio-transmitted to a high-end computer.

MEMS may have better power efficiency than that of CMOS electronics. Therefore, it might be desirable to use MEMS not only as sensors, but also as simple analog information processing units that could act on the information directly, e.g., removing noise, compensating for nonlinearities, performing analog Fourier transformations, etc., thereby leading to smart sensor technology. Even more interesting is the possibility to use MEMS for sophisticated information processing, such as pattern recognition, thereby eliminating the need for microcontrollers entirely.

In this paper we develop a theory to show that a network of coupled MEMS oscillators can function as a neurocomputer having oscillatory autocorrelative associative memory. This is based on our previous result that networks of arbitrary oscillators have associative memory when coupled appropriately [8], [9], [13]. The physical nature of the oscillators and the detailed

Manuscript received December 10, 1999; revised July 10, 2000. This paper was recommended by Associate Editor P. Szolgay.

F. C. Hoppensteadt is with the Center for Systems Science and Engineering, Arizona State University, Tempe, AZ 85287-7606 USA.

E. M. Izhikevich is with the Neurosciences Institute, San Diego, CA 92121 USA (e-mail: Eugene.Izhikevich@nsi.edu.).

Publisher Item Identifier S 1057-7122(01)01392-7. form of the equations describing their dynamics is not important for the theory. For example, networks of such diverse oscillators as phase-locked loops (PLLs) [6] and lasers [7] have identical neuro-computational properties. We show here the same for networks of MEMS oscillators.

We derive a canonical model describing nonlinear dynamics of a single MEMS oscillator in Section II, and consider a network of such oscillators in Section III. The derivation is valid only when the oscillators exhibit small amplitude periodic activity, which happens when damping becomes negative, the rest state becomes unstable, and an oscillation builds up. From a dynamical systems point of view this corresponds to an Andronov-Hopf bifurcation. To keep our exposition as simple as possible, we do not provide a detailed derivation of the canonical model, which is done in [9]-[11]. In Section IV we apply the theory to pattern recognition problem.

\section{A Single MEMS OSCILLATOR}

MEMS oscillators are being developed to provide miniature substitutes for crystal oscillators in wireless communication and signal processing applications [17]. Detailed information about their electro-mechanics can be found in [16] and [18].

The most important part of a MEMS oscillator is a polycrystalline silicon micromechanical resonator (MEMS resonator) similar to the one depicted in Fig. 1. A typical resonator has a comb-like geometry, which enhances its performance. The resonator consists of a spring-like shuttle that is anchored at its center to the ground plane; see Fig. 2. It supports two combs of fingers and allows them to oscillate in the $x$-direction. A dc-bias voltage $V_{p}$ is applied to the resonator and its ground plane to excite and bias the device. As with a pendulum, the resonator has a fundamental resonance frequency. If an ac-excitation voltage $v$ having this frequency is applied to the comb transducers, the mechanical structure starts to vibrate in the $x$-direction, thereby generating a motional output current $i$, which is amplified by the current-to-voltage amplifier and fed back to the resonator. This positive feedback loop can destabilize the system and lead to sustained oscillations.

\section{A. Basic Equations}

The complete and accurate set of equations describing dynamics of a single MEMS resonator (let alone a network of thereof) is not known due to many implementation details. Following [16], we start from the Lienard equation, which describes motion of a nonlinear pendulum

$$
m \ddot{x}+f(x, \lambda) \dot{x}+g(x)=0
$$




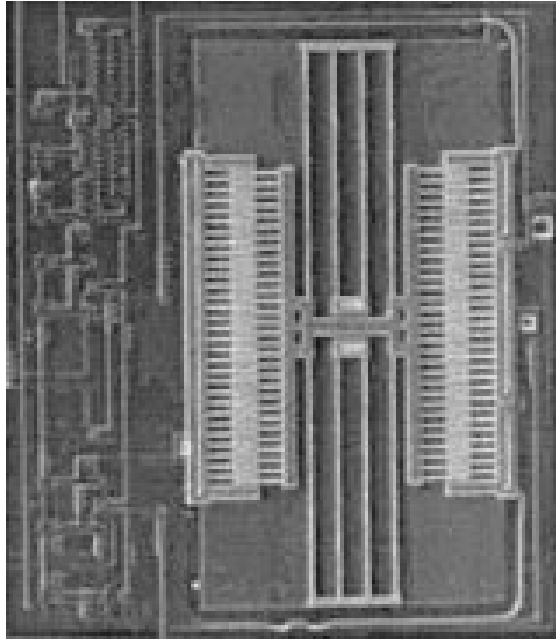

Fig. 1. A scanning-electron micrograph of a $16.5-\mathrm{KHz}$ folded-beam, capacitive-comb transduced CMOS micromechanical resonator, which occupies $230 \times 420-\mu \mathrm{m}^{2}$ (modified from [18]).

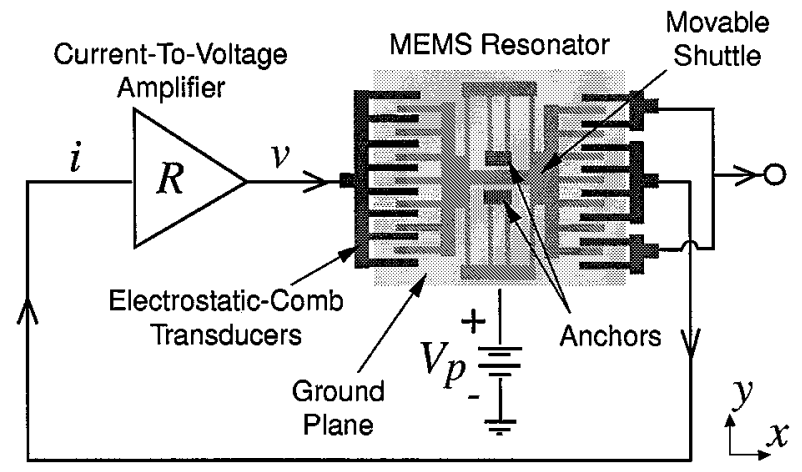

Fig. 2. System-level schematic of the MEMS oscillator, which consists of MEMS resonator (depicted in Fig. 1) and current-to-voltage amplifier that creates a positive feedback loop.

where variable $x$ denotes the displacement of the shuttle from the rest position $x=0$. The constant $m$ is its effective mass, and $f(x, \lambda)$ and $g(x)$ are the damping and stiffness functions, respectively. The former depends on the parameter $\lambda$, which can represent the dc-bias voltage $V_{p}$ applied to the oscillator, the gain of the sustained current-to-voltage amplifier, or any other physical parameter that affects the gain of the positive feedback loop.

The form of the damping function $f(x, \lambda)$ varies because it depends on details of the feedback loop, which may include an automatic level control circuitry that regulates the oscillation's amplitude. Damping at rest $x=0$ is positive when $\lambda$ is less than a certain threshold value $\lambda_{H}$, and negative otherwise. A typical example is the van der Pol damping, $f(x, \lambda)=x^{2}-\lambda$, with $\lambda_{H}=0$. While $\lambda$ increases through $\lambda_{H}$, the damping changes sign, the rest state $x=0$ becomes unstable, and the MEMS resonator begins small amplitude oscillation, which grows with $\lambda$. Such a transition from rest to oscillation corresponds to the supercritical Andronov-Hopf bifurcation, which is illustrated in Fig. 3.

The form of the stiffness function $g(x)$ depends on the geometry of the oscillator and the properties of its materials. If it is symmetric without defects, then $g(x)$ is an odd function, i.e.,
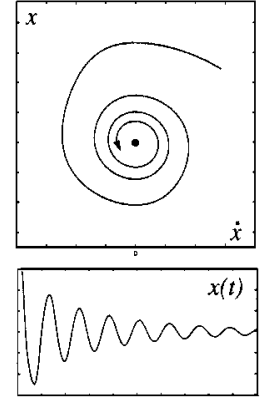

Positive Damping $f(0, \lambda)>0 \quad\left(\lambda<\lambda_{H}\right)$
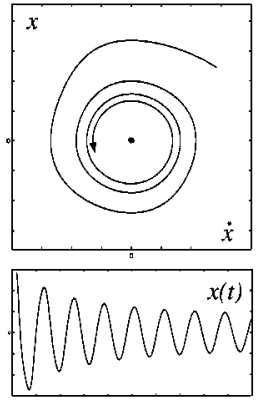

Zero Damping

$f(0, \lambda)=0 \quad\left(\lambda=\lambda_{H}\right)$
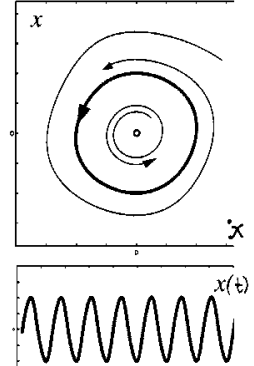

Negative Damping $f(0, \lambda)<0 \quad\left(\lambda>\lambda_{H}\right)$
Fig. 3. Andronov-Hopf bifurcation in MEMS oscillator (1) when the parameter $\lambda$ crosses the threshold value $\lambda_{H}$.

$g(-x)=-g(x)$. A typical example of this is the Duffing stiffness $g(x)=x+x^{3}$.

\section{B. Derivation of the Model}

The system spring constant

$$
k=g^{\prime}(0)>0
$$

plays an important role in determining the frequency of oscillation when $\lambda$ crosses the threshold value $\lambda_{H}$. Indeed, since $f\left(0, \lambda_{H}\right)=0$ and $g(x) \approx k x$ for small $x$, the Lienard equation (1) has the form

$$
m \ddot{x}+k x+\text { high-order terms }=0 .
$$

Neglecting the high-order terms results in linear harmonic oscillation with the frequency

$$
\omega=\sqrt{\frac{k}{m}} \text { (radians) }
$$

and an arbitrary amplitude. However, to find the amplitude and frequency of the nonlinear device, one needs to account for high-order terms, which we do next.

It is convenient to introduce a complex variable

$$
z=\dot{x}+\mathrm{i} \omega x \in \mathbb{C}
$$

that describes dynamics of the oscillator. One can easily check that (2) acquires a simple form

$$
\dot{z}=\mathrm{i} \omega z+\text { high-order terms. }
$$

Normal form theory [5], [9], [14] enables us to transform this equation into

$$
\dot{z}=(c+\mathrm{i} \omega) z+(a+\mathrm{i} b) z|z|^{2}
$$

where

$$
\begin{aligned}
& a=-\frac{f_{x x}^{\prime \prime}}{16 m \omega^{2}} \\
& b=\frac{3 m g^{\prime \prime \prime}-2\left(f_{x}^{\prime}\right)^{2}}{48 m^{2} \omega^{3}} \\
& c=-\frac{f_{\lambda}^{\prime}}{2 m}\left(\lambda-\lambda_{H}\right)
\end{aligned}
$$


and all derivatives are evaluated at the point $(x, \lambda)=\left(0, \lambda_{H}\right)$ (see [5, p. 155]). Converting (1) into the normal form (3) is standard in dynamical system theory. The supercritical Andronov-Hopf bifurcation occurs when $a<0$ and $c$ increases through the value 0 . This corresponds to $f_{x x}^{\prime \prime}>0$ and $\lambda$ crossing $\lambda_{H}$.

We emphasize that particular forms of the functions $f(x, \lambda)$ and $g(x)$, which depend on the resonator geometry, details of the feedback loop design, etc., do not affect the normal form (3), but only the values of coefficients $a, b$, and $c$. If the stiffness function $g(x)$ were not odd, e.g., due to material imperfections, and the damping function depended also on $\dot{x}$, then the oscillator dynamics could still be described by (3), but (4) and (5) would be more complicated

$$
\begin{aligned}
& a=\frac{f_{x}^{\prime} f_{\dot{x}}^{\prime}}{8 m^{2} \omega^{2}}+\frac{f_{x}^{\prime} g^{\prime \prime}}{16 m^{2} \omega^{4}}-\frac{f_{x x}^{\prime \prime}}{16 m \omega^{2}}-\frac{3 f_{\dot{x} \dot{x}}^{\prime \prime}}{16 m} \\
& b=\frac{g^{\prime \prime \prime}}{16 m \omega^{3}}-\frac{5\left(g^{\prime \prime}\right)^{2}}{48 m^{2} \omega^{5}}-\frac{\left(f_{x}^{\prime}\right)^{2}}{24 m^{2} \omega^{3}}-\frac{\left(f_{\dot{x}}^{\prime}\right)^{2}}{6 m^{2} \omega}-\frac{5 f_{\dot{x}}^{\prime} g^{\prime \prime}}{24 m^{2} \omega^{3}}
\end{aligned}
$$

while (6) would be unchanged.

\section{Analysis of the Model}

Systems (1) and (3) have equivalent dynamics for all $\lambda$ near $\lambda_{H}$. The latter system, however, has an advantage that all its parameters can be measured experimentally. To understand their meaning, we rewrite (3) in the polar coordinates

$$
z=r e^{i \vartheta}
$$

where $r=|z|$ is the amplitude and $\vartheta=\operatorname{Arg} z$ is the phase of oscillation (see [9, Chap. 10] for detailed discussion). Differentiating this equation with respect to $t$ gives

$$
\begin{aligned}
\dot{z} & =\dot{r} e^{\mathrm{i} \vartheta}+r e^{\mathrm{i} \vartheta} \mathrm{i} \dot{\vartheta} \\
& =(c+\mathrm{i} \omega) r e^{\mathrm{i} \vartheta}+(a+\mathrm{i} b) r^{3} e^{\mathrm{i} \vartheta} .
\end{aligned}
$$

Dividing the right-hand sides by $e^{\mathrm{i} \vartheta}$ yields

$$
\dot{r}+r \dot{\mathrm{i}}=(c+\mathrm{i} \omega) r+(a+\mathrm{i} b) r^{3}
$$

and separating the real and imaginary parts gives

$$
\begin{aligned}
& \dot{r}=c r+a r^{3} \\
& \dot{\vartheta}=\omega+b r^{2} .
\end{aligned}
$$

The first equation describes dynamics of the amplitude and the second of the phase.

When the parameter $\lambda$ is below the threshold value $\lambda_{H}$, the parameter $c$ defined in (6) is negative. Then the amplitude $r(t) \rightarrow 0$ exponentially, which corresponds to a damped oscillation. When the parameter is above the threshold, $c>0$ and

$$
r(t) \rightarrow \sqrt{\frac{c}{|a|}} .
$$

Thus, measuring the amplitude of a sustained oscillation, one can estimate the ratio $c / a$.

Equation (8) describes how the amplitude affects the frequency, which is $\omega+b r^{2}$. One can determine $b$ experimentally by measuring the frequency shift for various $r$. The condition $b=0$ may be desirable in applications requiring stable frequency. From (5) it follows that $b=0$ when the damping and stiffness satisfy

$$
3 m g^{\prime \prime \prime}=2\left(f_{x}^{\prime}\right)^{2}
$$

at $x=0$ and $\lambda=\lambda_{H}$. This equation is useful for designing the automatic level control circuitry for high- $Q$ oscillators.

\section{A NETWORK OF MEMS OsCILLATORS}

Now we consider a network of $n$ coupled MEMS oscillators

$$
m \ddot{x}_{i}+f\left(x_{i}, \lambda_{i}\right) \dot{x}_{i}+g\left(x_{i}\right)=\sum_{j=1}^{n}\left(p_{i j} \dot{x}_{j}+k_{i j} x_{j}\right)
$$

for $i=1, \ldots, n$. Here $p_{i j}$ and $k_{i j}$ are conductances and mechanical spring constants, which describe the strength of electrical and mechanical connections from the $j$ th to the $i$ th oscillator, respectively. If the oscillators are coupled via soft springs, i.e., via low stiffness beams, then (10) needs additional equations for beam dynamics.

System (10) has been investigated in [9, Chaps. 5, 10] and in [10] and [11]. There one can find details of the reduction of (10) to the canonical model

$$
\dot{z}_{i}=\left(c_{i}+\mathrm{i} \omega\right) z_{i}+(a+\mathrm{i} b) z_{i}\left|z_{i}\right|^{2}+\sum_{j=1}^{n} c_{i j} z_{j}
$$

where $z_{i}=\dot{x}_{i}+\mathrm{i} \omega x_{i}$

$$
c_{i j}=\frac{1}{2 m} p_{i j}-\frac{\mathrm{i}}{2 m \omega} k_{i j}
$$

and the other parameters were defined in the previous section.

\section{A. Polar Coordinates}

Let $c_{i j}=s_{i j} e^{\mathrm{i} \psi_{i j}}$, where $s_{i j}=\left|c_{i j}\right|$ is the effective strength of coupling and $\psi_{i j}=\operatorname{Arg} c_{i j}$ is the natural phase shift. The canonical model (11) has the following form in polar coordinates [10], [11]:

$$
\begin{aligned}
& \dot{r}_{i}=c_{i} r+a r_{i}^{3}+\sum_{j=1}^{n} s_{i j} r_{j} \cos \left(\vartheta_{j}+\psi_{i j}-\vartheta_{i}\right) \\
& \dot{\vartheta}_{i}=\omega+b r_{i}^{2}+\frac{1}{r_{i}} \sum_{j=1}^{n} s_{i j} r_{j} \sin \left(\vartheta_{j}+\psi_{i j}-\vartheta_{i}\right) .
\end{aligned}
$$

If all oscillators have positive amplitudes and $|a|$ is much larger that the effective strength of connections $\left|s_{i j}\right|$, then one can discard (13) and study dynamics in the frequency domain using

$$
\dot{\vartheta}_{i}=\omega_{i}+\sum_{j=1}^{n} w_{i j} \sin \left(\vartheta_{j}+\psi_{i j}-\vartheta_{i}\right)
$$

where $\omega_{i}=\omega+b c_{i} /|a|$ and $w_{i j}=s_{i j} \sqrt{c_{j} / c_{i}}$. The major advantage of this system is that one can easily study synchronization [12] and other nonlinear locking properties, which we do next. 

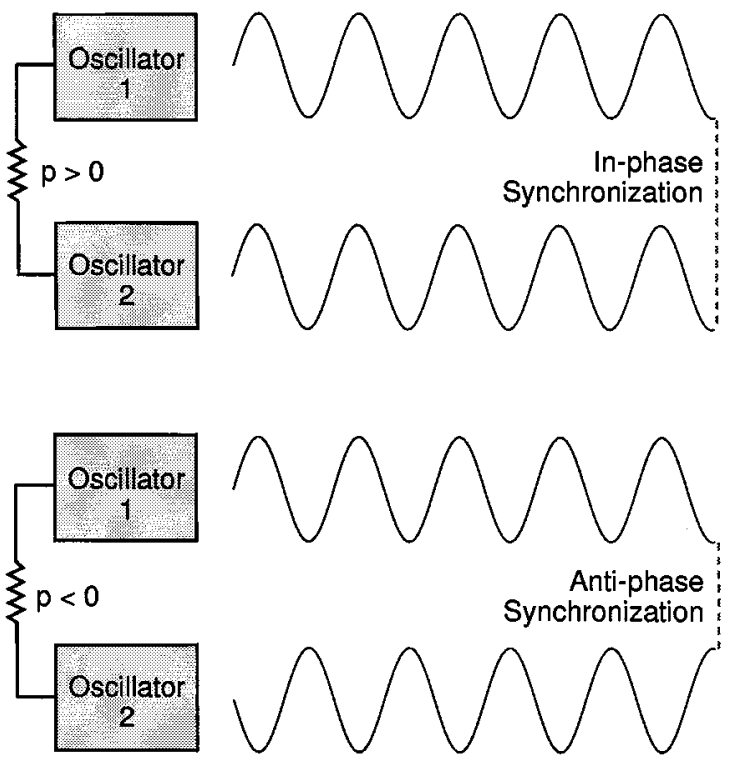

Fig. 4. The type of locking of identical MEMS oscillators depends on the sign of coefficient of electrical coupling $p=p_{12}=p_{21}$ in (10). Top: $p>0$ leads to in-phase synchronization. Bottom: $p<0$ leads to anti-phase synchronization.

\section{B. Two Oscillators}

Consider two identical oscillators having symmetrical electrical couplings of strength $p=p_{12}=p_{21}$. If $p>0$, then from (12) and the fact $k_{i j}=0$ it follows that $c_{12}=c_{21}$ are real and positive. Hence $\psi_{12}=\psi_{21}=0$, and the oscillators synchronize in-phase; that is, they oscillate with equal amplitudes and zero phase difference; see the top of Fig. 4. Indeed, we can consider the phase difference variable $\chi=\vartheta_{2}-\vartheta_{1}$ and note that

$\dot{\chi}=\dot{\vartheta}_{2}-\dot{\vartheta}_{1}=s_{21} \sin (-\chi)-s_{12} \sin (\chi)=-\left(s_{12}+s_{21}\right) \sin \chi$

Therefore, the phase difference $\chi(t) \rightarrow 0$. If $p<0$, then $\psi_{12}=$ $\psi_{21}=\pi$, and the oscillators synchronize anti-phase; that is, with $\chi(t) \rightarrow \pi$, see the bottom of Fig. 4 . Variations in oscillator parameters or coupling would slightly shift the phase difference.

Mechanical coupling affects the imaginary part of the connection coefficients $c_{i j}$ through the coefficients $k_{i j}$ and makes locking dynamics more complicated. The case of two identical oscillators was investigated in [9, Sect. 10.3], and it was shown that there can be stable in-phase or anti-phase synchronizations, or even their co-existence, depending on the values of the parameters.

\section{Many Oscillators}

Analysis of a network of $n>2$ oscillators is a daunting problem unless certain restrictions are imposed on the parameters. In this section we assume that $b=0$ so that the frequency does not depend on the amplitude, at least in the leading order. In this case we have a rather pleasant result that lays the basis for oscillatory neurocomputing using MEMS oscillators: If the oscillators have symmetrical electrical coupling, then the network always converges to an oscillatory phase-locked pattern; that is, the oscillators have equal frequencies and constant, but not necessarily identical, phases. There could be many such

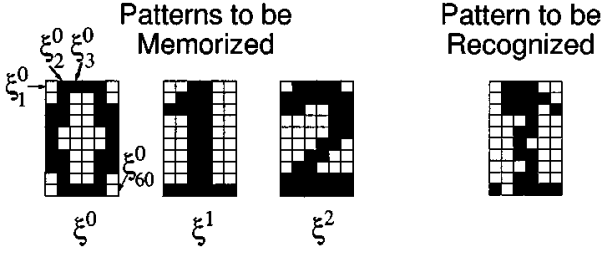

Fig. 5. Three patterns to be memorized by a network of MEMS oscillators.

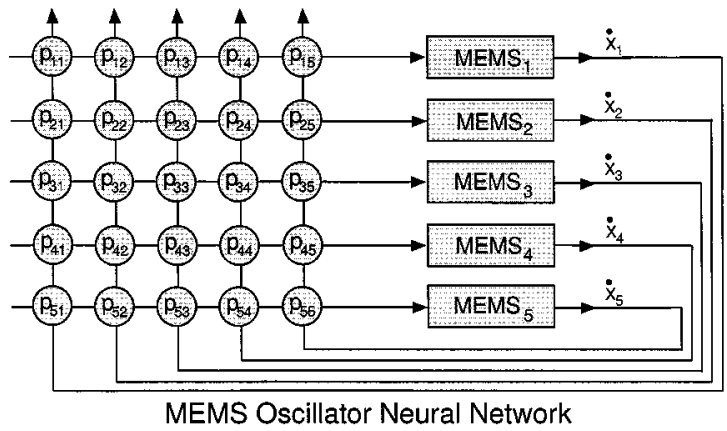

Fig. 6. A fully connected network of MEMS oscillators has oscillatory associative memory. Constants $p_{i, j}$ are conductances.

phase-locked patterns corresponding to many memorized images. The proof of this can be found in [11] and in [9, Sect. 10.4]. It follows from the existence of an energy function

$$
E(z)=-\sum_{i=1}^{n}\left(c_{i}\left|z_{i}\right|^{2}-\frac{a}{2}\left|z_{i}\right|^{4}\right)-\sum_{i, j=1}^{n} c_{i j} \bar{z}_{i} z_{j}
$$

for the canonical model (11).

\section{PATTERN RECOGNITION USING MEMS OSCILLATORS}

A natural approach to pattern recognition is to use artificial neural networks having associative memory [2]. Although that promised more than it could accomplish, it still remains attractive since artificial neural networks possess an advantageous computational property, parallelism. Each network consists of simple, slow unreliable units, i.e., artificial neurons, processing information in parallel. An enormous computational speed can be achieved when the number of neurons is large.

Artificial neurons are usually sigmoidal units described by a system similar to

$$
\dot{x}_{i}=-x_{i}+S\left(a_{i}+\sum_{j=1}^{n} c_{i j} x_{j}\right)
$$

where $S(y)=1 /\left(1+e^{-y}\right)$ is the sigmoidal function, $a_{i}$ is the threshold parameter, and $c_{i j}$ is a connection coefficient from $j$ th to $i$ th neuron. The dynamics of the network depends on the connection matrix $C=\left(c_{i j}\right)$ that retains the memory of "memorized" patterns. Below we show how one can use MEMS oscillators to build a device that implements various neural network architectures.

\section{A. Hopfield-Grossberg Networks}

Suppose we are given a set of binary patterns to be memorized

$$
\xi^{k}=\left(\xi_{1}^{k}, \xi_{2}^{k}, \ldots, \xi_{n}^{k}\right), \quad \xi_{i}^{k}= \pm 1, k=0, \ldots, m
$$




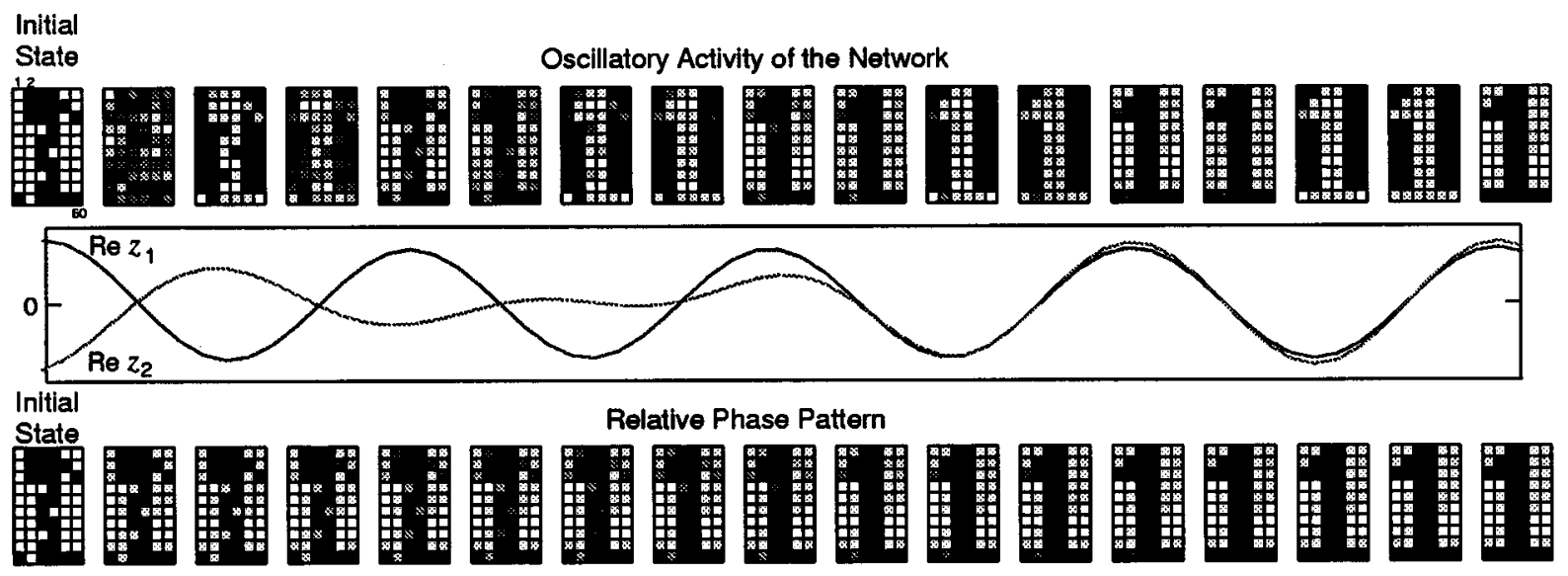

Fig. 7. Pattern recognition by a network of MEMS oscillators. Shown are simulations of the canonical model (11) with $a=-1, b=0, c=1$, and $\omega=8$. Hebbian learning rule (16) is used to "memorize" patterns from Fig. 5.

where $\xi_{i}^{k}=-1(+1)$ denotes a black (white) pixel; see Fig. 5. The case of continuous (graded) patterns corresponds to complex-valued vectors having $\xi_{i}^{k}=e^{\mathrm{i} \varphi_{i}^{k}}$, and it is considered elsewhere [9], [13].

One can use a Hebbian learning rule

$$
c_{i j}=\frac{1}{n} \sum_{k=0}^{m} \xi_{i}^{k} \xi_{j}^{k}
$$

to form the connection matrix $C=\left(c_{i j}\right)$, which is always symmetric, i.e., $c_{i j}=s_{i j}$ for all $i$ and $j$. The Cohen-Grossberg convergence theorem [4] and a number of other results show that the memorized images become attractors of the sigmoidal artificial neural network (15) provided that the number of patterns is not very large in comparison with the size of the network. For example, if an initial state of the network corresponds to a distorted pattern $\xi^{1}$, then the network converges to an attractor corresponding to nondistorted pattern $\xi^{1}$ so that $x_{i} \approx \xi_{i}^{1}$ for all $i$. (A negative pattern, $-\xi^{1}$, is also an attractor). Such networks are said to have autocorrelative association memory. We refer to them as being Hopfield-Grossberg networks.

\section{B. MEMS Neurocomputer}

The convergence result discussed in Section III-C claims that a network of MEMS oscillators described by (10) and depicted in Fig. 6 with the Hebbian learning rule (16) has associative memory similar to that of Hopfield-Grossberg networks, but a greater memory capacity [1], [13]. There is one important distinction though: The attractors are not equilibria (fixed points), but phase-locked oscillatory states with phase relations corresponding to the memorized patterns $\xi^{k}$. For example, the attractor corresponding to the memorized pattern $\xi^{1}$ has $i$ th and $j$ th oscillators synchronized in-phase when the $i$ th and the $j$ th pixels have the same color, i.e., when $\xi_{i}^{1}=\xi_{j}^{1}$, and synchronized anti-phase when the pixels have different colors. Notice that the problem of negative images is not an issue in oscillatory neural networks, since both $\xi^{1}$ and its negative, $-\xi^{1}$, define the same pattern of phase relations. Moreover, the network oscillates between $\xi^{1}$ and $-\xi^{1}$ periodically as we see in Fig. 7 .

We use the canonical model (11) with $n=60$ oscillators to illustrate the pattern recognition process. First, three patterns,

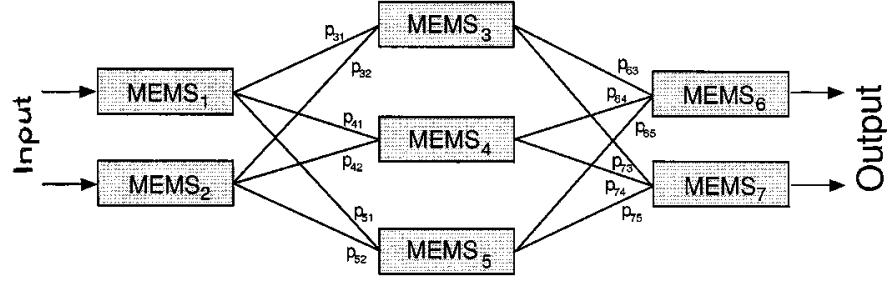

Fig. 8. A two-layer perceptron consisting of electrically coupled MEMS oscillators.

"0," "1," and "2" from Fig. 5 are "memorized" using the Hebbian rule (16), which results in a symmetric matrix of connections $C=\left(c_{i j}\right)$ [the same rule would be used to determine the coefficients of electrical coupling, $p_{i j}$, in (10)]. Then we distort pattern " 1 " and present it to the network as an initial state. (A detailed discussion of the initialization procedure can be found in [7] and [8].) To observe dynamics of the associative memory recall we plot as pixels real parts of variables $z_{i}$ at the top of Fig. 7 . Since the network has rhythmic dynamics, the picture alternates between positive and negative image. However, during recall the oscillators change their phases so that the correct pattern "1" is recovered. In the middle of Fig. 7 we plot $\operatorname{Re} z_{1}(t)$ and $\operatorname{Re} z_{2}(t)$ for comparison. Since the first and the second pixels have different colors initially, the oscillators $z_{1}$ and $z_{2}$ have an anti-phase relation at $t=0$. During the associative memory recall they synchronize in-phase so that the pixels acquire the same color eventually. At the bottom of Fig. 7 we depict the evolution of relative phase relations between the oscillators. One can clearly see how pattern " 1 " is gradually recovered.

This pattern recognition process is relatively insensitive to the parameters of the canonical model (11) as long as $b=0$ and the connections are symmetric. Since the canonical model (11) and the original system (10) have equivalent dynamics, they have identical neuro-computational properties. In particular, one can obtain Fig. 7 by simulating (10) directly.

\section{DISCUSSION}

We derive here a canonical model describing dynamics of a network of coupled MEMS oscillators near the onset of periodic activity. We use the model to study the theoretical possi- 
bility of using MEMS oscillators to build an oscillatory neurocomputer having autocorrelative associative memory similar to the one in Hopfield-Grossberg artificial neural networks. Incidentally, the patterns depicted in Fig. 5 have also been used to test oscillatory neuro-computational properties of networks of lasers [7] and phase-locked loops [6]. In all cases we obtained similar results, which confirms the theoretical prediction [8], [9] that neurocomputing is a universal property of all coupled oscillators regardless of their physical nature [13].

One can also use MEMS oscillators to build other signal processing units, such as multilayer perceptrons depicted in Fig. 8. Development of an appropriate "error backpropagation" algorithm to "teach" such an oscillatory perceptron is an interesting open problem.

MEMS oscillatory neurocomputers can be combined with MEMS sensors and actuators to create "super-smart" matter. Moreover, one can envisage an architecture in which the strength of interconnections between MEMS oscillators is not pre-computed using the Hebbian or some other learning rule, but depends on the current environment so that the network rewires itself dynamically every time the environment changes.

\section{REFERENCES}

[1] T. Aoyagi, "Network of neural oscillators for retrieving phase information,", Phys. Rev. Lett., vol. 74, pp. 4075-4078, 1995.

[2] M. A. Arbib, Brain Theory and Neural Networks. Cambridge, MA: MIT Press, 1995

[3] D. G. Aronson, G. B. Ermentrout, and N. Kopell, "Amplitude response of coupled oscillators," Physica D, vol. 41, pp. 403-449, 1990.

[4] M.A. Cohen and S. Grossberg, "Absolute stability of global pattern formation and parallel memory storage by competitive neural networks," IEEE Trans. Syst., Man, Cybern., vol. SMC-13, pp. 815-826, 1983.

[5] J. Guckenheimer and D. Holmes, Nonlinear Oscillations, Dynamical Systems, and Bifurcations of Vector Fields. New York: Springer-Verlag, 1983.

[6] F. C. Hoppensteadt and E. M. Izhikevich, "Pattern recognition via synchronization in phase-locked loop neural networks," IEEE Trans. Neural Networks, vol. 11, pp. 734-738, 2000.

[7] - "Synchronization of laser oscillators, associative memory, and optical neurocomputing," Phys. Rev. E., vol. 62, pp. 4010-4013, 2000

[8] — "Oscillatory neurocomputers with dynamic connectivity," Phys. Rev. Lett., vol. 82, pp. 2983-2986, 1999.

[9] - Weakly Connected Neural Networks. New York: SpringerVerlag, 1997

[10] _-, "Synaptic organizations and dynamical properties of weakly connected neural oscillators: I. Analysis of canonical model," Biological Cybern., vol. 75, pp. 117-127, 1996

[11] — "Synaptic organizations and dynamical properties of weakly connected neural oscillators. II. Learning of phase information," Biological Cybern., vol. 75, pp. 129-135, 1996.
[12] E. M. Izhikevich, "Weakly pulse-coupled oscillators, FM interactions, synchronization, and oscillatory associative memory," IEEE Trans. Neural Networks, vol. 10, pp. 508-526, 1999.

[13] _ _ "Computing with oscillators," Neural Networks, submitted for publication

[14] Y. Kuznetsov, Elements of Applied Bifurcation Theory. New York: Springer-Verlag, 1995

[15] A. Mason, N. Yazdi, A. V. Chavan, K. Najafi, and K. D. Wise, "A generic multielement microsystem for portable wireless applications," Proc. IEEE, vol. 86, pp. 1733-1746, 1998.

[16] C. T.-C. Nguyen and R. T. Howe, "An integrated CMOS micromechanical resonator high-Q oscillator," IEEE J. Solid-State Circuits, vol. 34, pp. 440-455, 1999.

[17] C. T.-C. Nguyen, "Micromechanical devices for wireless communications," in Proc. 1998 IEEE Int. Workshop Micro Electro Mechanical Systems, Heidelberg, Germany, Jan. 25-29, 1998, pp. 1-7.

[18] _ "Micromechanical resonators for oscillators and filters," in Proc. 1995 IEEE Int. Ultrasonics Symp., Seattle, WA, Nov. 7-10, 1995, pp. 489-499.

[19] N. Yazdi, F. Ayazi, and K. Najafi, "Micromachined inertial sensors," Proc. IEEE, vol. 86, pp. 1640-1659, 1998.

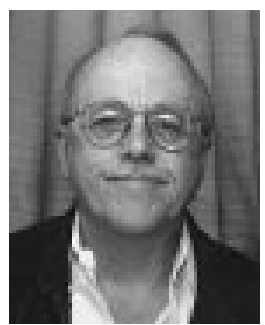

Frank C. Hoppensteadt (M'89) was born in Oak Park, IL in 1938. He received the Ph.D. degree in mathematics from the University of Wisconsin, Madison.

$\mathrm{He}$ is currently Director of the Regent's Center in system science and engineering research, Professor of mathematics and Professor of electrical engineering at Arizona State University. He has published 12 books and numerous articles about perturbation methods for differential equations and about mathematical problems in the life sciences. His present interests are in mathematical neuroscience, biotechnology and random perturbations of dynamical systems.

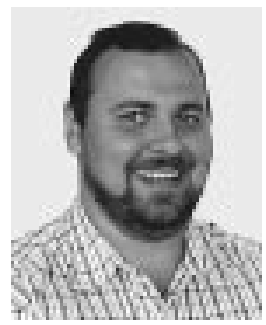

Eugene M. Izhikevich was born in Moscow, Russia, in 1967. He received the Master's degree in applied mathematics and computer sciences from Lomonosov Moscow State University, Moscow, Russia in 1992, and the Ph.D. degree in mathematics from Michigan State University, MI, in 1996.

$\mathrm{He}$ is currently an Associate Fellow in theoretical neurobiology at The Neurosciences Institute, San Diego, CA. His research interests include nonlinear dynamics of biological neurons and neural systems.

Dr. Izhikevich is a member of the International Neural Network Society. He won the SIAM Student Award for the best student paper in applied mathematics in 1995. 